\title{
Chapter 1 \\ Nuclear Magnetic Resonance Study of Cs Adsorption onto Clay Minerals
}

\author{
Yomei Tokuda, Yutaro Norikawa, Hirokazu Masai, Yoshikatsu Ueda, \\ Naoto Nihei, Shigeto Fujimura, and Yuji Ono
}

\begin{abstract}
The release of radioactive cesium into the environment in the aftermath of disasters such as the Fukushima Daiichi disaster poses a great health risk, particularly since cesium easily spreads in nature. In this context, we perform solidstate nuclear magnetic resonance (NMR) experiments to study $\mathrm{Cs}^{+}$ions adsorbed by clay minerals to analyze their local structure. The NMR spectra show two kinds of peaks corresponding to the clays (illite and kaolinite) after immersion in $\mathrm{CsCl}$ aqueous solution; the peak at $-30 \mathrm{ppm}$ is assigned to $\mathrm{Cs}^{+}$on the clay surface while that at $-100 \mathrm{ppm}$ is assigned to $\mathrm{Cs}^{+}$in the silicate sheet in the clay crystal. This result is consistent with the fact that $\mathrm{Cs}^{+}$with smaller coordination number yields a small field shift in the NMR spectra. Moreover, after immersion in $\mathrm{KCl}$ aqueous solution, these peaks disappear in the NMR spectra, thereby indicating that our assignment is reasonable. This is because $\mathrm{Cs}^{+}$on the clay surface and in the silicate sheet is easily subject to ion exchange by $\mathrm{K}^{+}$. We believe that our findings will contribute to a better understanding of the pathway through which Cs transfers from the soil to plants and also to the recovery of the agriculture in Fukushima.
\end{abstract}

Y. Tokuda $(\square) \bullet$ Y. Norikawa $\bullet$ H. Masai

Institute for Chemical Research, Kyoto University, Gokasho, Uji, Kyoto 611-0011, Japan

e-mail: tokuda@noncry.kuicr.kyoto-u.ac.jp

Y. Ueda

Research Institute for Sustainable Humanosphere, Kyoto University, Gokasho, Uji,

Kyoto 611-0011, Japan

N. Nihei

Graduate School of Agricultural and Life Sciences, The University of Tokyo, 1-1-1, Yayoi, Bunkyo-ku, Tokyo, Japan

S. Fujimura

NARO Tohoku Agricultural Research Center, 50 Harajukuminami, Arai, Fukushima-shi,

Fukushima 960-2156, Japan

Y. Ono

Fukushima Agricultural Technology Centre, 116, Shimonakamichi, Takakura, Hiwadamachi,

Koriyama, Fukushima 963-0531, Japan

T. Takahashi (ed.), Radiological Issues for Fukushima's Revitalized Future,

DOI 10.1007/978-4-431-55848-4_1 
Keywords Radioactive cesium $\bullet$ Cs adsorption by soil $~$ Cs NMR spectra - Kaolinite • Illite

\subsection{Introduction}

The occurrence of the Tohoku earthquake on March 11, 2011, led to the meltdown of the Fukushima Daiichi Nuclear Power Station in Japan. The accident released several kinds of radioactive elements such as ${ }^{90} \mathrm{Sr},{ }^{134} \mathrm{Cs},{ }^{137} \mathrm{Cs}$, and ${ }^{131} \mathrm{I}$ into the environment. Human exposure to ${ }^{137} \mathrm{Cs}$ is a health risk because of its long half-life [1]. The element ${ }^{137} \mathrm{Cs}$ is mostly stabilized in the soil while small quantities are absorbed by plants such as rice [2]. In this manner, ${ }^{137} \mathrm{Cs}$ seeps into the food chain, leading to its internal exposure in humans and animals. In order to avoid internal exposure, it is important to understand the mechanism underlying its transfer from the soil to plants. Recently, it has been reported that the Cs transfer coefficient exhibits variation even for plants grown in soils with similar levels of radioactivity $[3,4]$. Moreover, artificially added ${ }^{137} \mathrm{Cs}$ can be more easily absorbed by plants than stable Cs in the soil [5]. Thus, we have considered that one of the reasons for this phenomenon is the varied ways in which Cs (i.e., different Cs states) is adsorbed onto clay minerals in the soils.

The clay minerals that stabilize Cs include 1:1-type layer silicates and 2:1-type layer silicates [6]. These silicates stabilize Cs on the surface and silicate sheets because of their negative charges. In particular, in the case of 2:1-type layer silicates, Cs is strongly adsorbed at frayed edge sites (FESs) [7-10]. In this context, it is necessary to understand the stabilization mechanism to analyze the structure of Cs in the clay surface, silicate sheet, and FESs.

The concentration of $\mathrm{Cs}$ in the environment is of the order of parts per billion (ppb) or parts per trillion (ppt). Thus, very fine measurement techniques are required to measure such minute quantities and obtain their structural information. The technique of X-ray absorption fine structure (XAFS) spectroscopy is suitable for such measurements $[11,12]$. However, the technique of XAFS requires synchrotron radiation, and hence, the method cannot be used in the laboratory. Therefore, another complementary method is required.

In this context, solid-state nuclear magnetic resonance (NMR) has been used to analyze the local structure of Cs in crystals and conventional glasses [13]. As regards the NMR spectra of cesium silicate crystals, $\mathrm{Cs}^{+}$ions with large coordination numbers (CNs) such as $\mathrm{Cs}_{6} \mathrm{Si}_{10} \mathrm{O}_{23}$ exhibit a large field shift while those with smaller $\mathrm{CNs}\left(\mathrm{Cs}_{2} \mathrm{Si}_{2} \mathrm{O}_{5}\right)$ exhibit a small field shift. In addition, the same relationship also holds for Cs present in mixed alkali silicate glasses. This relationship can be used to study the local structure of Cs in clay minerals. Moreover, solid-state NMR of clay minerals can be utilized to distinguish Cs on the surface and within silicate sheets [14-17]. In this study, we discuss the structure of Cs adsorbed onto two kinds of clay minerals (kaolinite as 1:1-type layer silicates and illite as 1:2-type layer silicates) by using solid state NMR together with XAFS spectroscopy. 


\subsection{Experimental}

\subsubsection{Sample Preparation}

Kaolinite (Wako Chemicals), illite (G-O networks), ${ }^{133} \mathrm{CsCl}$ (Wako Chemicals), $\mathrm{KCl}$ (Wako Chemicals), and ultrapure water (Wako Chemicals) were used as received in our experiments. First, $5 \mathrm{~g}$ of illite was immersed in $50 \mathrm{~mL}$ of $0.01 \mathrm{M} \mathrm{CsCl}$ aqueous solution over time periods of 1 day, 1 month, 6 months, and 2 years. After immersion, the illite samples were separated by centrifugation. Next, $50 \mathrm{~mL}$ of ultrapure water was added to each illite sample followed by centrifugal separation. This washing process was performed twice. After washing, the illites were dried overnight at $40{ }^{\circ} \mathrm{C}$. We referred to the various samples as illite_1d, illite_1m, illite_6m, and illite_2y depending on their immersion periods of 1 day, 1 month, 6 months, and 2 years, respectively. For comparison purposes, kaolinite was also immersed in $50 \mathrm{~mL}$ of $0.01 \mathrm{M} \mathrm{CsCl}$ aqueous solution over 1 day, 1 month, and 6 months. The kaolinites were also washed using the abovementioned washing process. Following the nomenclature used for the illite samples, we named the kaolinite samples as kaolinite_1d, kaolinite_1m, and kaolinite_6m.

In order to remove the $\mathrm{Cs}$ adsorbed onto illite, $1 \mathrm{~g}$ of the sample illites_2y was immersed in $50 \mathrm{~mL}$ of $0.01 \mathrm{KCl}$ aqueous solution for $2 \mathrm{~h}$ and 2 days. These illites were also washed as per the abovementioned washing process. These "re-ion-exchanged" samples were referred to as illite_2y_KCl2h and illite_2y_KCl2d.

Further, pristine samples of illite and kaolinite were also analyzed. Table 1.1 lists all the analyzed samples along with the corresponding experimental conditions.

Table 1.1 Sample notations of clay minerals used in this study

\begin{tabular}{l|l|l}
\hline Notation & Period of immersion in $\mathrm{CsCl}(\mathrm{aq})$ & $\begin{array}{l}\text { Period of immersion in } \mathrm{KCl}(\mathrm{aq}) \\
\text { after immersion in } \mathrm{CsCl}(\mathrm{aq})\end{array}$ \\
\hline illite_prisitine & - & - \\
\hline illite_1d & 1 day & - \\
\hline illite_6m & 6 months & - \\
\hline illite_2y & 2 years & - \\
\hline illite_2y_KCl2h & 2 years & $2 \mathrm{~h}$ \\
\hline illite_2y_KCl2d & 2 years & 2 days \\
\hline kaolinite_pristine & - & - \\
\hline kaolinite_1d & 1 day & - \\
\hline kaolinite_6m & 6 months & - \\
\hline
\end{tabular}




\subsection{Structure Analyses}

The crystal structures were analyzed by powder X-ray diffraction (XRD) (RINT 2100, RIGAKU). We used a $\mathrm{Cu}$ X-ray source that was operated at $40 \mathrm{kV}$ and $40 \mathrm{~mA}$ via the conventional $2 \theta / \theta$ method. The diffractions were acquired at intervals of $0.02^{\circ}$. The extended X-ray absorption fine structure (EXAFS) spectra were obtained at the cesium K-absorption edge via the fluorescence method (BL14B2, SPring-8). The cumulated number of measurements was 40 . The XAFS spectra were analyzed by using ATHENA [18].

The solid-state ${ }^{133} \mathrm{Cs}$ NMR spectra of all the samples were acquired using a Chemagnetics CMX400 spectrometer utilizing a commercial probe $(4 \mathrm{~mm})$. The rotation speed was set to $10 \mathrm{kHz}$ with an accuracy $\pm 10 \mathrm{~Hz}$. At an external field of 9.4 T, the resonance frequency was set to about 103.7 MHz. For each measurement, the widths of the $90^{\circ}$ pulses were set to $2.2 \mu \mathrm{s}$. The spectra were obtained with a cycle time of $10 \mathrm{~s}$. The chemical shift reference was $1 \mathrm{~mol} / \mathrm{L} \mathrm{CsCl}$ aqueous solution, whose chemical shifts were set to $0 \mathrm{ppm}$.

\subsection{Results}

Figure 1.1 shows the XRD patterns of the illite_pristine, illite_6m, kaolinite_pristine, and kaolinite_6m samples. In the case of illite, the peak around $27^{\circ}$ shows a shift to a higher angle after immersion in $\mathrm{CsCl}$, thereby indicating a decrease in the lattice constant. On the other hand, for kaolinite, the peak around $27^{\circ}$ shifts to a lower angle, which indicates an increase in the lattice constant.

Figure 1.2 shows $\mathrm{k}^{2}$-weighted K-edge EXAFS spectra for the illite_2y and illite_2y_KCl2h samples. As previously reported for the radial distribution functions (RDFs), the first, second, and third peaks can be assigned to $\mathrm{Cs}-\mathrm{O}, \mathrm{Cs}-\mathrm{Si}$, and Cs-Cs, respectively $[19,20]$. However, we have not obtained the RDF in the present stage. We can just note that there is a little change in EXAFS spectra for the illite_2y and illite_2y_KCl2h samples.

Figure 1.3 shows the ${ }^{133}$ Cs NMR spectra of the illite and kaolinite samples after immersion in $\mathrm{CsCl}$ solution. The NMR spectra for all the illites exhibit peaks at around -30 and $-100 \mathrm{ppm}$. On the other hand, the NMR spectra for kaolinite_1d and _ $1 \mathrm{~m}$ exhibit only one peak at around $-30 \mathrm{ppm}$. The kaolinite_6m sample (which was immersed for a longer time) exhibits a clear peak at around $-30 \mathrm{ppm}$ and a small peak at around $-100 \mathrm{ppm}$.

The effect of re-ion-exchanging by $\mathrm{K}^{+}$on the NMR spectra was also studied (Fig. 1.4). The NMR peak for illite_2y_KCl2d vanished, although the peak at around $-30 \mathrm{ppm}$ was still observed for illite_2y_KCl2h. 
a

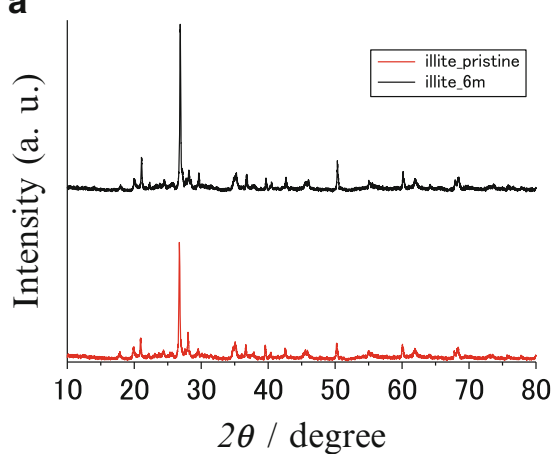

b

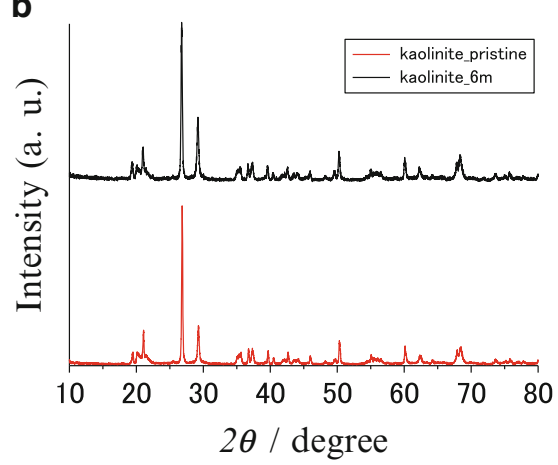

C

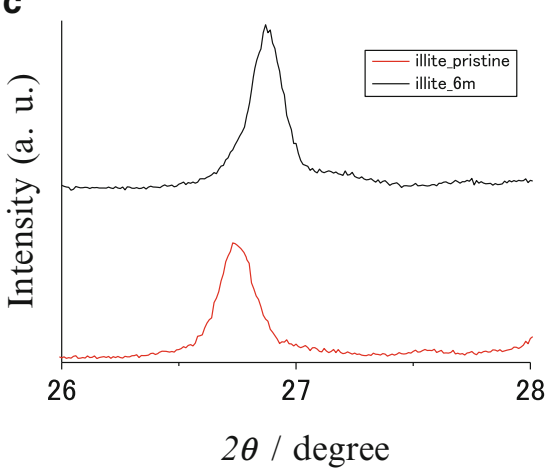

d

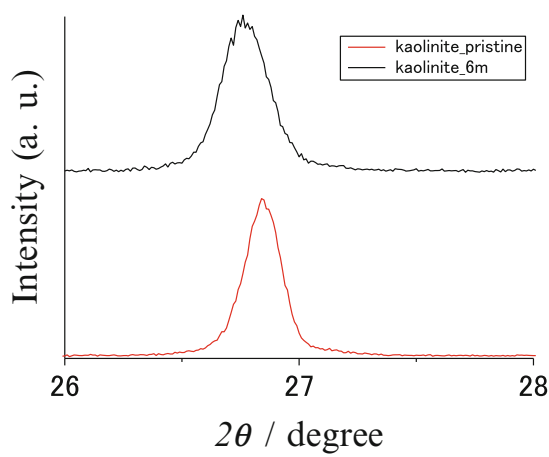

Fig. 1.1 XRD patterns of illite (a) as received and after immersion in $\mathrm{KCl}$ solution, (b) kaolinite as received and after immersion in $\mathrm{KCl}$ solution. Highest peaks around $27^{\circ}$ assigned to $\left(\begin{array}{lll}0 & 0 & 6\end{array}\right)$ are also shown as (c) and (d)

\subsection{Discussion}

After sample immersion in $\mathrm{CsCl}$ solution, the lattice constant change for illite is different from that for kaolinite, as shown in Fig. 1.1; the lattice constant of illite decreases, while that of kaolinite increases. This is because hydrated $\mathrm{K}^{+}$in the silicate sheet is replaced by $\mathrm{Cs}^{+}$in illite; on the other hand, in the case of kaolinite, a proton is replaced by $\mathrm{Cs}^{+}$. Alteration in the $\mathrm{k}^{2}$-weighted $\mathrm{K}$-edge EXAFS spectra for illite after immersion in $\mathrm{CsCl}$ solution as shown in Fig. 1.2 may support these observations.

As shown in Fig. 1.3, the NMR spectra of illite exhibit two peaks at -30 and -100 ppm, while those of kaolinite exhibit two peaks (one clear peak and one very small peak at -30 and -100 ppm, respectively). Kaolinite has a negative surface charge on the crystallite. Accordingly, the clear peak at $-30 \mathrm{ppm}$ can be assigned to the surface $\mathrm{Cs}^{+}$. In contrast, illite has a negative charge between silicate sheets. As a result, the peaks at $-100 \mathrm{ppm}$ can be assigned to $\mathrm{Cs}^{+}$in the silicate sheets. 


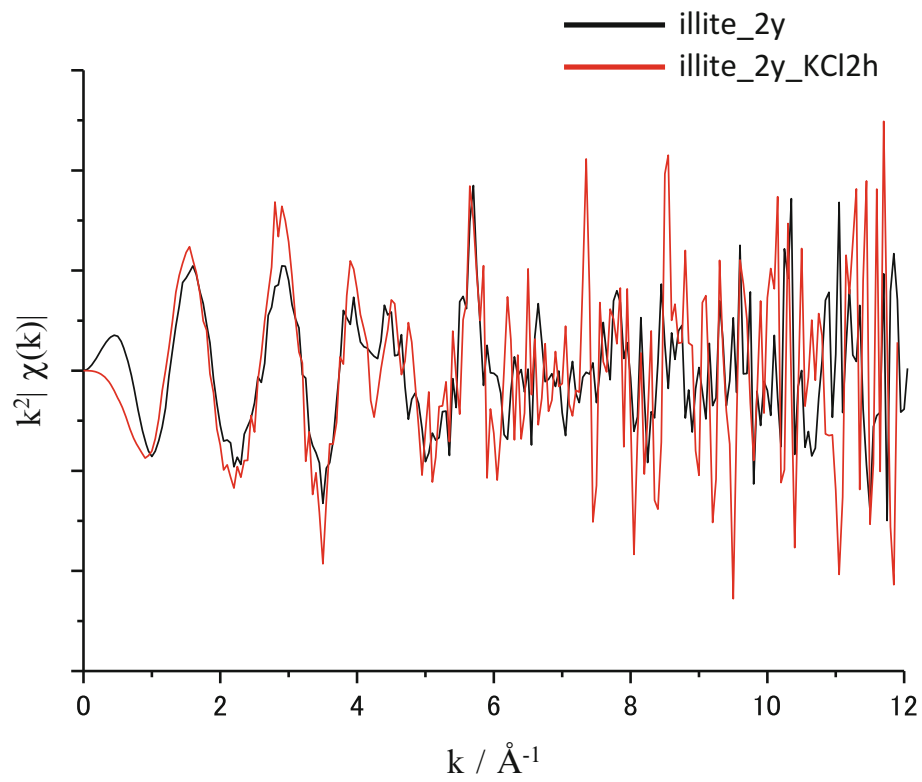

Fig. 1.2 $\mathrm{k}^{2}$-weighted K-edge EXAFS spectra for illite_2y and illite_2y_KCl2h

a

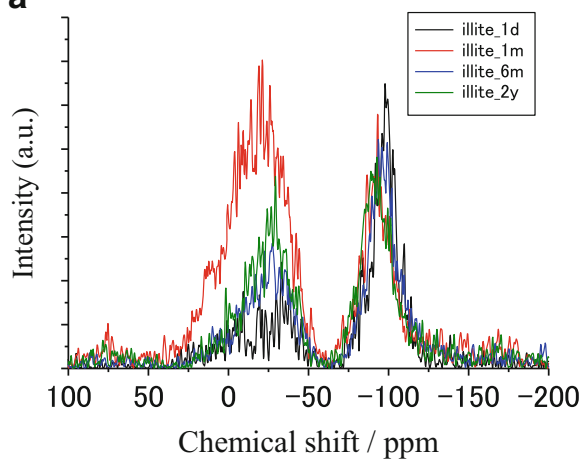

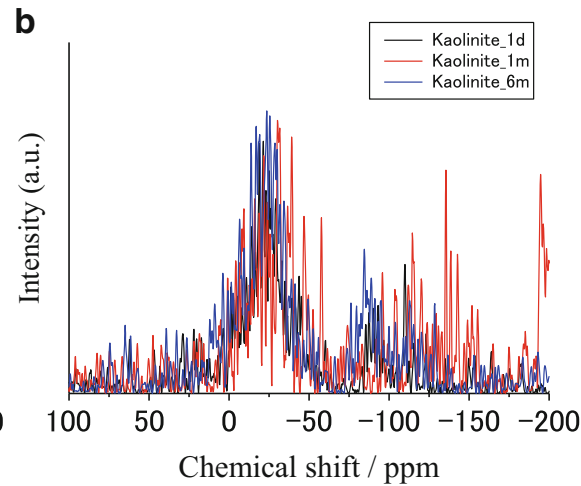

Fig. 1.3 NMR spectra of clays immersed in $\mathrm{CsCl}$ aqueous solution for several perids (a) illite, (b) kaolinite. The chemical shift reference is $\mathrm{CsCl}$ (aq)

These assignments agree well with the results of previous NMR experiments; $\mathrm{Cs}^{+}$ with larger $\mathrm{CN}$ values exhibits a large field shift, while that with smaller $\mathrm{CN}$ values exhibits a small field shift [13]. In another NMR experiment, there also existed two kinds of peaks for illite immersed in $\mathrm{CsCl}$ [14]. These results also support our assignment.

After re-ion-exchange (using $\mathrm{KCl}$ ) over a relatively long period ( 2 days) for illite (Fig. 1.4), no peak was observed in the NMR spectrum, thereby indicating that all 


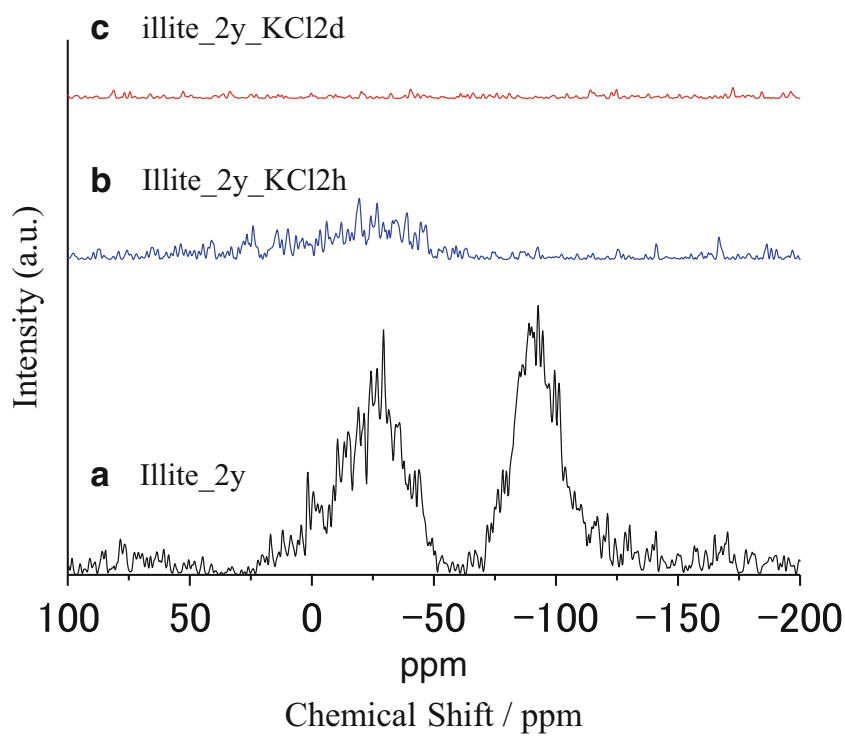

Fig. 1.4 NMR spectra of illite (a) immersion in $\mathrm{CsCl}$ solution for 2 years, (b) ion-exchanged illite by immersion in $\mathrm{KCl}$ for $2 \mathrm{~h}$, (c) ion-exchanged illite by immersion in $\mathrm{KCl}$ for 2 days. The chemical shift reference is $\mathrm{CsCl}(\mathrm{aq})$

the $\mathrm{Cs}^{+}$ions on the surface and in the silicate sheet had been replaced by $\mathrm{K}^{+}$. This result supports our assignment because $\mathrm{Cs}^{+}$on the clay surface and in the silicate sheet is easily ion-exchanged by $\mathrm{K}^{+}[6]$. This result also indicates that no FESs were observed in this experiment. One of the reasons for the absence of the FES signature is the low concentration of the total amount of $\mathrm{Cs}^{+}$. After re-ion-exchange for a short period of time $(2 \mathrm{~h})$, we observed only one peak at $-30 \mathrm{ppm}$. This result can be attributed to the fact that $\mathrm{Cs}^{+}$in the silicate sheet is more easily replaced by $\mathrm{K}^{+}$than that on the surface.

In conclusion, solid-state ${ }^{133} \mathrm{Cs} \mathrm{NMR}$ is useful for analyzing $\mathrm{Cs}^{+}$adsorbed onto clay minerals; this method can be used to distinguish $\mathrm{Cs}^{+}$sites in the clay minerals. In the near future, we plan to perform structure analysis of $\mathrm{Cs}^{+}$in soil in order to understand the mechanism of transfer of Cs from the soil to plants. We believe that this study will contribute to the recovery of agriculture in Fukushima in the near future.

\subsection{Conclusions}

We performed ${ }^{133}$ Cs NMR experiments in conjunction with XRD and EXAFS to analyze the adsorption of $\mathrm{Cs}^{+}$onto clay minerals such as illite and kaolinite. Our NMR results indicate that the observed peaks at -30 and $-100 \mathrm{ppm}$ can be assigned 
to $\mathrm{Cs}^{+}$on the surface and in the silicate sheet, respectively. After re-ion-exchange by using aqueous $\mathrm{KCl}$, the $\mathrm{Cs}^{+}$ions were replaced by $\mathrm{K}^{+}$ions. We believe that our findings will contribute to a better understanding of the mechanism of $\mathrm{Cs}$ transfers from the soil to plants. We plan to use the NMR method in our future studies on understanding of the mechanism of transfer of $\mathrm{Cs}^{+}$from the soil to plants.

Acknowledgments This work was supported by the Collaborative Research Program of Institute for Chemical Research, Kyoto University (No. 2015- 68, 70), Research Institute for Sustainable Humanosphere, Kyoto University, and Kureha Trading Co., Ltd. The synchrotron radiation experiments were performed at the BL14B2 beamline of the SPring-8 facility with the approval of the Japan Synchrotron Radiation Research Institute (JASRI) (Proposal No. 2015A1662). We would like to thank Kenji Hara, Hiroshi Goto and Hironori OFuchi for fruitful discussions.

Open Access This chapter is distributed under the terms of the Creative Commons Attribution Noncommercial License, which permits any noncommercial use, distribution, and reproduction in any medium, provided the original author(s) and source are credited.

\section{References}

1. IAEA (2006) Environmental consequences of the Chernobyl accident and their remediation: twenty years of experience. International Atomic Energy Agency, Vienna

2. Fujimura S, Muramatsu Y, Ohno T, Saitou M, Suzuki Y, Kobayashi T, Yoshioka K, Ueda Y (2015) Accumulation of (137)Cs by rice grown in four types of soil contaminated by the Fukushima Dai-ichi Nuclear Power Plant accident in 2011 and 2012. J Environ Radioact 140:59-64

3. Kato N, Kihou N, Fujimura S, Ikeba M, Miyazaki N, Saito Y, Eguchi T, Itoh S (2015) Potassium fertilizer and other materials as countermeasures to reduce radiocesium levels in rice: results of urgent experiments in 2011 responding to the Fukushima Daiichi Nuclear Power Plant accident. Soil Sci Plant Nutr 61:179-190

4. Ehlken S, Kirchner G (2002) Environmental processes affecting plant root uptake of radioactive trace elements and variability of transfer factor data: a review. J Environ Radioact 58:97-112

5. Tsukada H, Nakamura Y (1999) Transfer of ${ }^{137} \mathrm{Cs}$ and stable Cs from soil to potato in agricultural fields. Sci Total Environ 228:111-120

6. Yamaguchi N, Takata Y, Hayashi K, Ishikawa S, Kuramata M, Eguchi S, Yoshikawa S, Sakaguchi A, Asada K, Wagai R, Makino T, Akahane I, Hiradate S (2012) Behavior of radiocaesium in soil-plant systems and its controlling factor (in Japanese). Bull Nat Inst AgroEnviron Sci 31:75-129

7. Fuller AJ, Shaw S, Ward MB, Haigh SJ, Mosselmans JFW, Peacock CL, Stackhouse S, Dent AJ, Trivedi D, Burke IT (2015) Caesium incorporation and retention in illite interlayers. Appl Clay Sci 108:128-134

8. Okumura M, Nakamura H, Machida M (2014) First-principles studies of cesium adsorption to frayed edge sites of micaceous clay minerals. In: Abstract paper of the 248thAmericanchemicalsocietynationalmeeting\&exposition, San Francisco

9. Sato K, Fujimoto K, Dai W, Hunger M (2013) Molecular mechanism of heavily adhesive Cs: why radioactive Cs is not decontaminated from soil. J Phys Chem C 117:14075-14080

10. Cremers A, Elsen A, Depreter P, Maes A (1988) Quantitative-analysis of radiocesium retention in soils. Nature 335:247-249 
11. Fan Q, Yamaguchi N, Tanaka M, Tsukada H, Takahashi Y (2014) Relationship between the adsorption species of cesium and radiocesium interception potential in soils and minerals: an EXAFS study. J Environ Radioact 138:92-100

12. Bostick BC, Vairavamurthy MA, Karthikeyan KG, Chorover J (2002) Cesium adsorption on clay minerals: an EXAFS spectroscopic investigation. Environ Sci Technol 36:2670-2676

13. Minami T, Tokuda Y, Masai H, Ueda Y, Ono Y, Fujimura S, Yoko T (2014) Structural analysis of alkali cations in mixed alkali silicate glasses by ${ }^{23} \mathrm{Na}$ and ${ }^{133} \mathrm{Cs}$ MAS NMR. J Asian Ceram Soc 2:333-338

14. Kim Y, Cygan RT, Kirkpatrick RJ (1996) ${ }^{133}$ Cs NMR and XPS investigation of cesium adsorbed on clay minerals and related phases. Geochim Cosmochim Acta 60:1041-1052

15. Kim Y, Kirkpatrick RJ (1997) ${ }^{23} \mathrm{Na}$ and ${ }^{133} \mathrm{Cs}$ NMR study of cation adsorption on mineral surfaces: local environments, dynamics, and effects of mixed cations. Geochim Cosmochim Acta 61:5199-5208

16. Kim Y, Kirkpatrick RJ, Cygan RT (1996) ${ }^{133}$ Cs NMR study of cesium on the surfaces of kaolinite and illite. Geochim Cosmochim Acta 60:4059-4074

17. Maekawa A, Momoshima N, Sugihara S, Ohzawa R, Nakama A (2014) Analysis of ${ }^{134}$ Cs and ${ }^{137} \mathrm{Cs}$ distribution in soil of Fukushima prefecture and their specific adsorption on clay minerals. J Radioanal Nucl Chem 303:1485-1489

18. Ravel B, Newville M (2005) ATHENA, ARTEMIS, HEPHAESTUS: data analysis for X-ray absorption spectroscopy using IFEFFIT. J Synchrotron Radiat 12:537-541

19. Yaita T (2013) Interpretation of Cs adsorption behavior based on the EXAFS, TR-Dxafs, and STXM methods. Mineral Mag 77:2532

20. Yaita T, McKinley I (2013) Fundamental approaches toward development of radiocesium removal methods from soil and the other related materials, waste reduction and management optimization. http://fukushima.jaea.go.jp/initiatives/cat01/pdf00/07_Yaita.pdf 